\title{
Increased number of Judo therapy facilities in Japan and changes in their geographical distribution
}

\author{
Satoshi Inoue, Mutsuhiro Nakao ${ }^{*}$, Kyoko Nomura, Eiji Yano
}

\begin{abstract}
Background: Judo therapy is a well established Japanese co-medical profession specializing in outpatient manual treatment of fractures and sprains. Recently, the number of judo therapists has been rapidly increasing as a result of proliferation judo therapy academies. This study examines whether such rapid increases have improved geographical distribution of judo therapy facilities in Japan.

Methods: The number of judo therapy facilities and the population in each municipality were obtained from the Web yellow pages and from Japanese census data for 2004, 2006, and 2008, respectively. Lorenz curves and Gini indices were calculated to demonstrate distributions of judo therapy facilities per 100,000 people. A bootstrapped method was used to identify statistical significances of differences in Gini indices.

Results: In all municipalities, the mean numbers of judo therapy facilities per 100,000 people were 15.3 in 2004, 15.8 in 2006, and 17.6 in 2008. The Gini indices for judo therapy facilities nationally were 0.273 in 2004, 0.264 in 2006, and 0.264 in 2008. The numbers of judo therapy facilities increased significantly between 2006 and 2008 ( $p<$ $0.05)$ but the indices did not change significantly in the same period. The Gini indices for local towns and villages remained unchanged and were consistently higher $(p<0.05)$ than those in urban areas throughout the study periods.
\end{abstract}

Conclusion: Our results suggest that recent increases in the number of judo therapy facilities have not necessarily led to greater equality in their geographic distribution in terms of Gini indices.

\section{Background}

Judo therapy, originating in the Japanese martial art of judo, represents one of Japan's most unique and traditional co-medical professions [1]. Primarily, judo therapists manually treat sprains, bruises, soft tissue damage, fractures, and dislocations in their own offices. Since 1920, judo therapists have required state licensing. Following the 1998 court decision that rejected regulations promulgated by the Japanese Ministry of Health, Labour and Welfare limiting the total number of judo therapists [2], the number of academies training judo therapists increased from 14 to 70 . As a result of the increased number of academies, new graduates qualifying as judo therapists began to practice during the period from

\footnotetext{
* Correspondence: mnakao@med.teikyo-u.ac.jp

* Correspondence: mnakao@med.teikyo-u.ac.jp
Department of Hygiene and Public Health, Teikyo University School of Medicine, Tokyo, Japan
}

(c) 2011 Inoue et al; licensee BioMed Central Ltd. This is an Open Access article distributed under the terms of the Creative Commons Attribution License (http://creativecommons.org/licenses/by/2.0), which permits unrestricted use, distribution, and reproduction in any medium, provided the original work is properly cited. free access to healthcare service under universal coverage of health insurance in Japan; it has been achieved with relatively low cost (340 billion dollars annually for national health expenditure, 9\% of GDP). In general, under the Japanese Health Insurance System, judo therapists can operate their facilities independently, and total medical expenditures for judo therapy have been estimated at greater than 3 billion dollars annually [3]. Thus, the practice of judo therapy requires careful monitoring.

Judo therapists are expected to provide complementary and alternative medical treatments, particularly in areas with insufficient medical care services. In this sense, assessing the geographic distribution of judo therapists is important. If the increased number of judo therapists were concentrated primarily in urban areas, which also contain a relatively large number of 
orthopedists, the overlapping services provided by both professions might lead to conflicts between them. Some orthopedists have rumored that patients at their clinics are recruited by judo therapists. Clearly, for the current increased numbers of judo therapists to provide their services more equitably in terms of geographic distribution would be preferable, but no published studies have examined this possibility.

The Lorenz curve and Gini indices, originally used in economics research to assess income inequality $[4,5]$, were chosen to describe geographic distribution in this study because they can be used to summarize resource distribution on a formal, standardized scale from " 0 " (even distribution) to " 1 ' (greatest possible unevenness of distribution). Gini indices can provide a standardized basis on which to make judgments on the comparative degrees of geometric unevenness for different manpower resource pools at a point in time, or over a period of time [6,7]. The indices have frequently been used to study disproportionate distributions of health services, including those pertaining to the numbers of physicians and medical facilities [8-13]. For example, using Gini indices, Kobayashi studied the number of physicians in Japanese municipalities and reported the changes in geographical distribution before and after the increase in medical schools [10]. Several reports using Gini indices for the geographical density of physicians, especially practicing physicians and pediatricians, have been published since the mid-1990s [11-13]. To perform the same type of analysis to assess disproportionate distributions of judo therapy facilities, we developed our database of the number of judo therapy facilities in each Japanese municipality and have published two reports on the geographic density of judo therapists $[14,15]$. However, both studies were cross-sectional in study design and the geographic distribution of judo therapists was not examined over time.

Thus, the objective of this study involved examining recent changes in the geographic distribution of judo therapy facilities in relation to the increase in judo therapy academies in Japan. Assuming that the orthopedists have a primary right to be located where they are, and do not require redistribution themselves, we would like to determine if the expansion of judo therapy in Japan since 1998 has been equitable between urban and rural areas, rather than concentrating in the urban areas where orthopedists have already been established. The hypothesis of this study was that as the number of therapy facilities expanded, so a more equal pattern of provision would be observed. To test this hypothesis, the Gini index of the number of judo therapy facilities at each of the national and regional levels was used as the simple parameter for statistical analysis. Then the Gini indices and their 95\% confidence intervals of the number of judo therapy facilities were estimated at twoyear intervals during 2004 to 2008, when the number of judo therapy facilities rapidly increased, to compare between 2004 and 2006 and between 2006 and 2008.

\section{Methods}

\section{Number of judo therapy facilities}

The data set, developed from the NTT Internet Townpage Directory of Internet sites [16] in July 2004, 2006, and 2008, was composed of the number of judo therapy facilities in 3,218 municipalities throughout Japan. This method was validated in our previous studies [14,15]; the total number of judo therapy facilities obtained from the Townpage Directory $(=21,995)$ was close to the number of judo therapy facilities in all prefectures reporting health insurance payments $(=23,199)$ in 2002. The Internet search used "judo therapist" and the name of each municipality as keywords. Among the search results, only "Sekkotsu-in (facility for bone setting)" and "Seikotsu-in (osteopathy facility)" were selected because other facilities are prohibited from practicing by the Japanese Health Insurance System. This study uses the number of judo therapy facilities instead of the number of judo therapists because no information could be gathered about the latter.

\section{Population by municipality}

The population of each municipality was estimated from census data $[17,18]$, as described in detail in our previous study [15]; the data set can be obtained from the corresponding author upon e-mail request. The incorporated population in July 2008 was used for the analysis of the municipalities consolidated after 2004. The populations of certain major cities were too large for direct comparisons; in these cases, Tokubetsu-ku (specific wards) were used for purposes of comparison. In total, 1,921 municipalities were defined for use in the analysis.

\section{Analysis}

All statistical analyses were performed using the STATA (Ver.9 for Windows) and two-tailed $p$ values of less than 0.05 were regarded as statistically significant. Although there were several measurements to report the geographic distributions $[10,19]$, this study used the mean numbers of judo therapy facilities per 100,000 people with their Gini indices according to our previous studies $[14,15]$ and Japanese other studies $[6,10]$. The Gini indices were calculated based on Lorenz curve, and the procedure of the calculation was as follows. Municipalities were sorted by the number of judo therapy facilities per 100,000 people. Beginning with the municipality with the fewest judo therapy facilities, the $\mathrm{x}$ axis of the Lorenz represents the cumulative percentage of the population. The y axis represents the cumulative 
percentages of judo therapy facilities. Under conditions of complete equality, the cumulative curve would coincide with the $45^{\circ}$ diagonal line. Unequal distributions produce cumulative curves below the $45^{\circ}$ line. The Gini index is defined as the proportion of the area surrounded by the $45^{\circ}$ line and the Lorenz curve in relation to the area below the $45^{\circ}$ line; this index ranges from 0 to 1 , with higher values indicating larger geographical gaps. In the same way, the Gini indices per 100,000 people were calculated individually for urban areas $(=919)$ and towns/villages $(=1,002)$.

The Gini idex is originally a single value reflecting unequal distributions, but the confidence intervals (C.I.s) of the Gini index can be obtained by a bootstrapping procedure [20]. The bootstrap is a computer-intensive method that draws independent samples from the data and calculates the target statistic on each draw. The bootstrap procedure uses the observed data to estimate the theoretical and usually unknown distribution from which the data came $[20,21]$. Bootstrap samples of the same size as the original sample are repeatedly drawn by sampling with replacement from the observed data. Based on previous studies [22,23] a bootstrap method with 1,000-time randomizations was used to estimate $95 \%$ C.I.s of the Gini index. A replacement random sampling was performed 1,921 times from the original database of judo therapy facilities per 100,000 people, and the Gini index was estimated using the created new dataset. This procedure was repeated 1,000 times, and the confidence bands of the Gini index were constructed by multiplicatively expanding the $2.5 \%$ and 97.5\% points of quantile functions of the simulated data so that the bands have $95 \%$ simultaneous coverage over the range of the Gini index. The estimated Gini indices were compared between urban areas and towns/villages for each year. Annual differences in the Gini indices were calculated and the bootstrap method was applied to determine statistical significance [22,23].

\section{Results}

Table 1 shows the number of judo therapy facilities per 100,000 people and the estimated Gini index. Although the general population gradually decreased during the period studied, the number of judo therapy facilities consistently increased, resulting in an increase of the number of judo therapy facilities per 100,000 people. The number of judo therapy facilities in the entire country increased by $5.3 \%$ in 2006 and $8.3 \%$ in 2008 per 100,000 people compared to the values obtained in 2004 and 2006, respectively. This increase was significant only in 2008 , which posted the same increase in urban areas. In contrast, no significant changes were found for towns/villages in both 2006 and 2008.

Compared to the values in the previous 2 years, the degrees by which the Gini index changed (95\% C.I.) were $-0.009(-0.013,-0.006)$ in 2006 and 0.0005 $(-0.006,0.007)$ in 2008 in all areas; they were -0.010 $(-0.013,-0.007)$ in 2006 and $0.0006(-0.005,0.006)$ in 2008 in urban areas, and $-0.003(-0.009,0.003)$ in 2006

Table 1 The number of judo therapy (J.T.) facilities, their distribution per 100,000 people, and Gini indices in all areas, urban areas, and towns/villages in 2004, 2006, and 2008

\begin{tabular}{|c|c|c|c|}
\hline & 2004 & 2006 & 2008 \\
\hline \multicolumn{4}{|l|}{ Population $(\times 1000)$} \\
\hline All areas $(n=1,921)$ & 127,902 & 127,758 & 126,931 \\
\hline Urban areas $(\mathrm{n}=919)$ & 114,557 & 114,552 & 114,049 \\
\hline Town/villages $(n=1,002)$ & 13,345 & 13,206 & 12,882 \\
\hline \multicolumn{4}{|l|}{ Number of J.T. facilities } \\
\hline All areas & 22,774 & 23,996 & 25,989 \\
\hline Urban areas & 20,888 & 22,045 & 23,906 \\
\hline Towns/villages & 1,886 & 1,951 & 2,083 \\
\hline \multicolumn{4}{|c|}{ J.T. facilities per 100,000 population* } \\
\hline All areas & $15.3 \pm 11.8(14.7-15.7)$ & $15.8 \pm 12.2(15.3-16.4)$ & $17.6 \pm 14.0(17.0-18.2) \dagger$ \\
\hline Urban areas & $18.0 \pm 9.8(17.4-18.6)$ & $19.0 \pm 9.9(18.3-19.6)$ & $20.9 \pm 12.1(20.2-21.7) \dagger$ \\
\hline Towns/villages & $12.8 \pm 12.9(12.0-13.6)$ & $13.1 \pm 13.3(12.2-13.9)$ & $14.8 \pm 14.9(13.6-15.4)$ \\
\hline \multicolumn{4}{|l|}{ Mean Gini $(95 \% \text { C.I.) })^{*}$} \\
\hline All areas & $0.273(0.261-286)$ & $0.2640 .253-0.276) \dagger$ & $0.264(0.251-0.276)$ \\
\hline Urban areas & $0.258 .248-0.267)$ & $0.2470 .238-0.257) \dagger$ & $0.248(0.239-0.257)$ \\
\hline Towns/villages & $0.407(0.394-0.419)$ & $0.404(0.392-0.417)$ & $0.400(0.388-0.413)$ \\
\hline
\end{tabular}

Mean values \pm standard deviations (95\% confidence intervals, C.I.s) of J.T. facilities per 100,000 people are presented; the mean Gini indices (95\% C.I.s) of their distributions were estimated using the bootstrap method.

${ }^{\dagger}$ The differences in values were significant $(p<0.05$, two-tailed t-test) when compared to those in the previous 2 years. 
and $-0.004(-0.011,0.003)$ in 2008 in towns/villages. This indicates that the Gini index significantly decreased in 2006 but not in 2008 in all areas and in urban areas but that it did not decrease significantly in either 2006 or in 2008 in towns/villages The Gini index was statistically higher (all $p<0.05$ ) in urban areas than in towns/villages in 2004, 2006, and 2008. The Lorenz curves of judo therapy facilities per 100,000 people in 2008 are presented for all, urban, and town/village areas in Figure 1.

\section{Discussion}

The present study demonstrates that the density of judo therapy facilities per population unit has increased, irrespective of area, from 2004 to 2008 along with the increase in the absolute number of judo therapy facilities. In particular, the extent to which judo therapy facilities per population unit increased in 2008 was statistically significant and higher than that in 2006 by a factor of more than 1.5. However, this increase in 2008 did not reflect equality in the geographic distribution of judo therapy facilities according to the Gini index. Rather, it reflects a trend toward increased geographical gaps between urban areas and the rest of Japan in 2008.

These findings are important because under the current laws and regulations governing medical delivery systems, the Japanese government cannot intervene in choices about where medical practitioners practice. For example, Japan's physician manpower policy during the

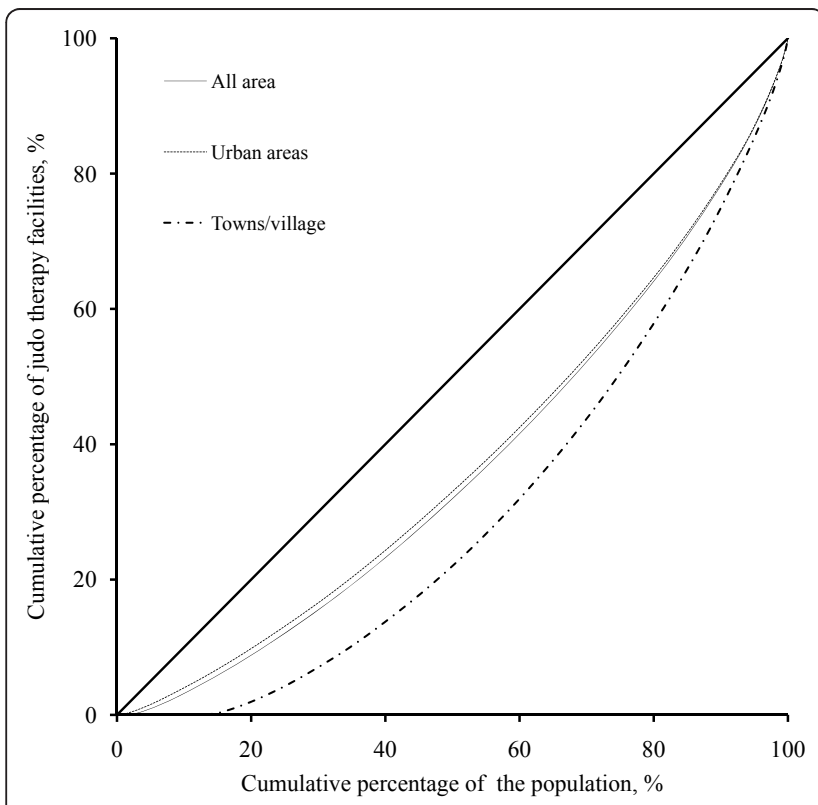

Figure 1 Lorenz curves of distributions of judo therapy facilities in all areas, urban areas, and towns/villages in 2008. The $45^{\circ}$ diagonal line represents a completely even distribution. Unequal distributions produce cumulative curves below the $45^{\circ}$ line. 1970s involved increasing the number of medical students and medical schools from 65 to 79 to address the shortage and maldistribution of physicians resulting in communities without doctors. The number of newly certificated physicians increased from approximately 4,000 to 8,000 per year by the mid-1980s. However, the inequality in physician distribution did not improve between 1980 and 1990 according to a previous Japanese study [10], suggesting that simply increasing the supply of medical providers does not constitute an advisable health policy. Rather, a policy that alleviates the maldistribution of medical providers should be developed. In the present study, the Gini indices significantly decreased in all areas and in urban areas only in 2006, even though the extent to which judo therapy facilities per population unit increased during this year was lower than that during 2008. Because the number of judo therapy facilities per population unit has remained at greater than 20 per 100,000 in urban areas since 2008, it seems clear that the rapid and substantial growth in the number of qualified judo therapists might not naturally match the geographic distribution of the need for judo therapy facilities.

In the present study, national data were not used for two reasons, even though all judo therapists are required to report to the designated public health center. First, the statistics on judo therapists are organized according to each public health center but not according to each municipality [24]. Second, these data were not completely accurate insofar as they might have included defunct businesses because reporting closures of these facilities is not required $[14,15]$. We finally decided to gather information about judo therapy facilities from the Townpage of each municipality, and the number obtained via this method was within $5 \%$ of the number of judo therapy facilities in all prefectures reporting health insurance payments. The number of facilities may represent a good surrogate for the number of therapists because in most cases, each facility contains only one practicing judo therapist. Using registration data obtained from the Japanese Judo Therapists' Association [25], we estimated that an average of 1.09 therapists worked in each facility during the period studied.

Before making remarks, several limitations should be noted. First of all, this study is not an analysis of the economics of service provision, but a use of a particular descriptive technology in assessing distributions of judo therapy facilities. Spatial inequality matters are largely due to the time price associated with any health facility, as well as other economic phenomenon including spatial access to health facilities [26,27]. Because geographical distribution of medical service provider directly relates to urgent need of human life in the community, distribution per se should be analyzed apart from economics. Financial aspects of Judo therapy facilities are important 
but beyond our scope requiring totally different data set and method for analysis. Thus the results of timechanges in geographic distribution of judo therapy facilities were simply shown in this study. In the future a variety of economic factors need to be considered to interpret our results before approving or opposing health-care policy for the number of judo therapy facilities. Second, this study is limited by its use of a municipality-based method to determine the number of judo therapy facilities and residents; the scale and nature of the facilities could not be assessed. These were grouped data, and the possible effects of 'ecological fallacy' should also be firmly considered for the interpretation of the results [28]. Also, the number of judo therapy facilities was divided by 100,000 people as the only indicator of 'need'. This was because the national data of health-care facilities have usually been published as a unit of per 100,000 residences in Japan, but we should bear in mind that different 'needs' indicators normally produce different inequality estimates: all of which have implications for health policy and planning competing health priorities. Third, the effects of unions of municipalities on the Gini indices should be considered. The Gini index is itself affected by the number of subjects analyzed $[17,18]$, and the indices for small towns and villages are smaller when such towns or villages are combined into larger cities. However, the number of united cities, towns, and villages was limited $(=27)$ from 2006 to 2008 and does not appear to account for changes in the geographical differences characterizing Japan in 2006. Fourth, four-year study period was relatively short to observe the change of distribution of judo therapy facilities. However, we were specifically interested in the change of distribution of judo therapy facilities from the start of drastic change of graduates qualifying as judo therapists (i.e., the years 2004 to 2006) in this study, and we recognize that the future study should be continued to observe the distribution of judo therapy facilities.

In spite of these limitations, we demonstrated that judo therapy facilities are widely but unevenly distributed. We suggest that recent increases in the number of judo therapy facilities do not necessarily lead to amelioration of inequalities in their geographic distribution. A large portion of elderly individuals live in nonurban areas, and this fact seems to reinforce the need for judo therapy in such areas [29]. Geographic distributions of health services are affected by forces of demand and supply, and in the future we would like to assess the needs for judo therapy and the number of qualified judo therapists comprehensively in all regions before considering intervention plans to motivate judo therapists to practice in the underserved local areas.

\section{Conclusions}

The numbers of judo therapy facilities increased significantly between 2006 and 2008 in Japan, but the Gini indices did not change significantly in the same period. The Gini indices for local towns and villages remained unchanged and were consistently higher than those in urban areas throughout the study periods. These results suggest that recent increases in the number of judo therapy facilities have not necessarily led to greater equality in their geographic distribution in terms of Gini indices.

\section{Acknowledgements}

This research was supported in part by Grant-in-Aid for Scientific Research (2010) (No. 10103152) from the Ministry of Health, Labour and Welfare, Japan.

\section{Authors' contributions}

SI collected the data, performed statistical analysis, and wrote the manuscript. MN and EY have made substantial contributions to conception and design, and have been involved in drafting and revising the manuscript. KN made substantial contributions to analysis and interpretation of data. All authors read and approved the final manuscript.

\section{Competing interests}

The authors declare that they have no competing interests.

Received: 17 September 2010 Accepted: 28 February 2011

Published: 28 February 2011

\section{References}

1. Japanese Ministry of Internal Affairs and Communications: Judo Therapist Act. [http://www.houko.com/00/01/S45/019.HTM], (in Japanese).

2. Fukuoka District Court: Legal report on the affair of regulation of the establishment of new schools for judo therapist training in 1998.(in Japanese) [http://www.courts.go.jp/hanrei/pdf/ AFC4CEDD28B71BE849256D41000B08F7.pdf].

3. Japanese Ministry of Health, Labour Welfare: The costs of judo therapy, massage therapy, acupuncture, and moxibustion. [http://search.e-gov.go, .jp/servlet/Organization?class=1050\&objcd=100495\&dispgrp=0170], (in Japanese).

4. Gastwirth JL: The estimation of the Lorenz curve and Gini index. Review of Economics and Statistics 1972, 54(3):306-316.

5. Slottje DJ: Research on economic inequality. Greenwich, Conn: JAI Press. New York; 19988.

6. Kim J, Miyamoto S, Shimizu H: Difference in floor areas of welfare facilities per student on the National University campuses. J Archit Plann Envioron Eng AlJ 1995, 469:97-105, (in Japanese).

7. Jahan S, Oda T: Distribution of public facilities in Dhaka, Bangladesh: a spatial analysis. Bulletin of the Faculty of Human Development 2000, 7(2):865-874.

8. Morrow JS: Toward a more normative assessment of maldistribution: the Gini index. Inquiry 1977, 14:278-292.

9. Yang BM, Huh J: Physician distribution and health manpower policy in Korea. Asia Pac J Public Health 1989, 3:68-85.

10. Kobayashi Y, Takaki H: Geographic distribution of physicians in Japan. Lancet 1992, 340:1391-1393.

11. Chang RK, Halfon N: Geographic distribution of pediatricians in the United States: an analysis of the fifty states and Washington, DC. Pediatrics 1997, 100:172-179.

12. Gravelle $H$, Sutton M: Inequality in the geographical distribution of general practitioners in England and Wales 1974-1995. J Health Serv Res Policy 2001, 6:6-13.

13. Kokaze K, Yoshida Y, Sekine Y, Teruya K, Takeda N, Uchida Y, et al: Trends in the geographic and age distribution of obstetrician-gynecologists and 
pediatricians in contrast to the decrease in the number of children in Japan. Jpn J Hyg Human Ecol 2001, 67:291-304, (in Japanese).

14. Inoue S, Karita K, Yano E: Geographical distribution of judo therapists and orthopedists in Japan. J Manipulative Physiol Ther 2005, 28:253-258.

15. Inoue S, Nomura K, Yano E: Geographical distribution of Judo therapist facilities and orthopedic facilities in Japan. Jpn J Pub Health 2008, 55:375-380, (in Japanese).

16. NTT Internet Townpage. [http://itp.ne.jp/servlet/jp.ne.itp.sear.SCMSVTop], (in Japanese).

17. Statistics Bureau, Japanese Ministry of Public Management, Home Affairs, Posts and Telecommunications: 2000 Population Census. Tokyo: Japanese Statistical Association; 2000, (in Japanese).

18. Tokyo Metropolitan Government Bureau Of General Affairs: Statistics of the population in Tokyo. [http://www.toukei.metro.tokyo.jp/jsuikei/2008/ js087a0000.xls], (in Japanese).

19. Williams RF, Doessel DP: Private psychiatry and Medicare: regional equality of access in Australia? Journal of Mental Health 2009, 18(3):242-252.

20. Efron B: The Jackknife, Bootstrap the and Other Resampling Plans. The Society for Industrial and Applied Mathematics. Philadelphia, Pennsylvania; 1982.

21. Meyer JS, Ingersoll CG, McDonald LL, Boyce MS: Estimating uncertainty in population growth rate: jackknife vs. bootstrap techniques. Ecology 1986, 67:1156-1166.

22. Davison AC, Hinkley DV: Bootstrap Methods and Their Application. New York: Cambridge University Press; 1997.

23. Joseph G, Reza M, Efstathia B: The use of the Lorenz curve, Gini index and related measures of relative inequality and uniformity in securities law. Int J Statistics 2005, 63:451-469.

24. Japanese Ministry of Health, Labour and Welfare: Report on Public Health Administration and Services 2006. Tokyo: Kousei Toukei Kyoukai; 2007, (in Japanese).

25. Japanese Judo Therapists Association: Report of Judo Therapy 2003.Edited by: Hara T. Tokyo: Taiyo Graphic; 2003:199, (in Japanese).

26. Williams RF, Doessel DP: Measuring inequality: tools and an illustration. Int J Equity Health 2006, 5:5.

27. Goddard M, Gravelle H, Hole A, Marini G: Where did all the GPs go? Increasing supply and geographical equity in England and Scotland. $J$ Health Serv Res Policy 2010, 15(1):28-35.

28. Takeuchi T, Nakao M, Nishikitani M, Yano E: Stress perception and social indicators for low back, shoulder and joint pains in Japan: national surveys in 1995 and 2001. Tohoku J Exp Med 2004, 203(3):195-204.

29. Nishikitani M, Inoue S, Yano E: Competition or complement: relationship between judo therapists and physicians for elderly patients with musculoskeletal disease. Environ Health Prev Med 2008, 13:123-129.

Pre-publication history

The pre-publication history for this paper can be accessed here: http://www.biomedcentral.com/1472-6963/11/48/prepub

doi:10.1186/1472-6963-11-48

Cite this article as: Inoue et al.: Increased number of Judo therapy facilities in Japan and changes in their geographical distribution. BMC Health Services Research 2011 11:48.

\section{Submit your next manuscript to BioMed Central and take full advantage of:}

- Convenient online submission

- Thorough peer review

- No space constraints or color figure charges

- Immediate publication on acceptance

- Inclusion in PubMed, CAS, Scopus and Google Scholar

- Research which is freely available for redistribution

Submit your manuscript at www.biomedcentral.com/submit
Biomed Central 